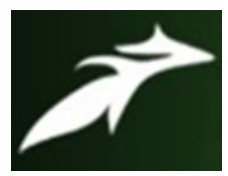

KOLA TIRUPATHI RAO et al, International Journal of Advances in Agricultural Science and Technology,

Vol.7 Issue.11, November-2020, pg. 45-54

ISSN: 2348-1358

Impact Factor: 6.057

NAAS Rating: 3.77

\title{
IMPACT OF DRIP IRRIGATION ON VEGETABLE CROP PRODUCTION ON ITS BENEFICIARIES IN GAJAPATHINAGARAM BLOCK OF VIZIANAGARAM DISTRICT ANDHRA PRADESH
}

\author{
KOLA TIRUPATHI RAO ${ }^{1}$; DR. DIPAK KUMAR BOSE ${ }^{2}$; DR. JAHANARA ${ }^{3}$ \\ MSc Scholar; Associate Professor; HOD \\ Department of Agricultural Extension \& Communication \\ Sam Higginbottom University of Agricultural Technology \& Sciences, Prayagraj (211007) \\ DOI: 10.47856/IJAAST.2020.v07i11.007
}

\begin{abstract}
Drip irrigation systems have the highest potential water application efficiency of the irrigation system used in commercial vegetable production. Drip irrigation is a tool to reduce water use, increase fertilizer efficiency and improve profit, while simultaneously reducing the potential risk to the environment due to enrichment of surface and groundwater. This research was conducted to evaluate the crop production in vegetable crops; the experiment should be conducted in the dry season to evaluate the effectiveness of drip irrigation on traditional vegetable production. Hence the present study was undertaken to find out the knowledge level of the drip irrigation farmers towards improved drip irrigation system technology with respect of socio-economic profile of the farmer of Gajapathinagaram block of vizianagaram district Andhra Pradesh. The study revealed that most of the respondents $(53.33 \%)$ had medium level of knowledge followed by low (11.67\%) and high $(35 \%) \&$ respondents $(40 \%)$ had High level of adoption followed by medium $(38.33 \%)$ and low (21.67\%).
\end{abstract}

KEYWORDS: Drip irrigation system, Knowledge, Association.

\section{INTRODUCTION:}

Drip irrigation provides slow, even application of low-pressure water to soil and plants using plastic tubing placed in or near the plants' root zone. It is an alternative to sprinkler or furrow methods of irrigating crops. Drip irrigation can be used for crops with high or low water demands. It provides sufficient base information for a grower to decide whether to further investigate the use of drip irrigation for their vegetable production operations. The involvement of farmers in the drip adopters are above $60 \%$. The origins of drip irrigation can be traced back to the ancient Egyptians, who in the $6^{\text {th }}$ millennium BCE developed the technique known as "flood irrigation" by creating a network of canals to channel water from the Nile to their gardens. Modern drip irrigation uses water-dispersing tapes, tubes and nozzles to deliver water drop by drop to the exact location of individual plants 


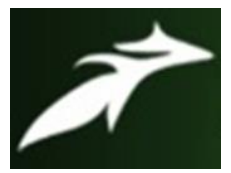

KOLA TIRUPATHI RAO et al, International Journal of Advances in Agricultural Science and Technology, Vol.7 Issue.11, November-2020, pg. 45-54

ISSN: 2348-1358

Impact Factor: 6.057

NAAS Rating: 3.77

in the field. This modern approach depends entirely on the development of suitable plastics for the technology involved. Older approaches that were not so efficient did use similar ideas. The basic concept of releasing less water in the right place dates back thousands of years.

\section{RESEARCH METHODOLOGY:}

Ex-post facto research design was used for the present study. Ex-post - facto research design is an inquiry in which the researcher does not have direct control of independent variable because their manifestations occurred and they cannot be manipulated. Influences about relations among variable are made without intervention from concomitant variation of independent and dependent variable. The state of Andhra Pradesh is divided into 13 administrative districts spread across two unofficial regions- Coastal Andhra and Rayalaseema. Coastal Andhra is divided into 9 districts. Out of these, Vizianagaram District is purposively selected for this study. Because the Drip irrigation system implementation in this area. APMIP (Andhra Pradesh Micro Irrigation Project) is going on this Vizianagaram district. In Vizianagaram district comprises of 34 blocks, Gajapathinagaram block was selected purposively because the block has sufficient number of vegetable growers using both drip and non-drip irrigation technology. Gajapathinagaram block comprises of 32 villages, out of which 20 villages have both drip and nondrip irrigation system. Out of these 32 villages, 6 villages were selected on the basis of crop grown by the selected respondents. Two types of farmers were selected in equal number from the each selected village who were using drip irrigation technology and other methods of irrigation except drip. The size of sample was 120 i.e. 60 vegetable growers using drip irrigation technology and 60 vegetable growers using non-drip irrigation technology. The data collected through interview schedule were transferred on the master sheet to describe characteristics of the respondents. For various items, frequencies were counted and percentage was calculated. To interpret the results and to show the relationship between independent variable and dependent variables, Mean, Frequency, Percentage, Coefficient correlation was followed. 


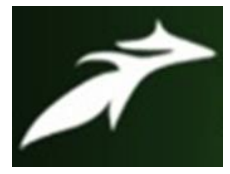

KOLA TIRUPATHI RAO et al, International Journal of Advances in Agricultural Science and Technology, Vol.7 Issue.11, November-2020, pg. 45-54

ISSN: 2348-1358 Impact Factor: 6.057 NAAS Rating: 3.77

RESULTS AND DISCUSSION:

To determine and compare the Socio-economic profile of beneficiaries and non-beneficiaries.

Table no:1 Showing Age wise Distribution of Beneficiaries and Non-Beneficiaries.

\begin{tabular}{|c|l|c|c|c|c|}
\hline \multirow{2}{*}{$\begin{array}{c}\text { S.N } \\
\mathbf{O}\end{array}$} & \multirow{2}{*}{ AGE } & \multicolumn{2}{|c|}{ BENEFICIARIES } & \multicolumn{2}{c|}{ NON BENEFICIARIES } \\
\cline { 3 - 5 } & & FREQUENCY & PERCENTAGE & FREQUENCY & PERCENTAGE \\
\hline 1 & YOUNG AGE 20-35 & 22.00 & 36.67 & 30.00 & 50.00 \\
\hline 2 & MIDDLE AGE 36-50 & 32.00 & 53.33 & 29.00 & 48.34 \\
\hline 3 & OLD AGE > 50 & 6.00 & 10.00 & 1.00 & 1.66 \\
\hline & TOTAL & 60.00 & 100.00 & 60.00 & 100.00 \\
\hline
\end{tabular}

Table no:2 Showing Education wise Distribution of Beneficiaries and Non-Beneficiaries.

\begin{tabular}{|c|c|c|c|c|c|}
\hline \multirow[t]{2}{*}{ S.NO } & \multirow[t]{2}{*}{ EDUCATION } & \multicolumn{2}{|c|}{ BENEFICIARIES } & \multicolumn{2}{|c|}{ NON BENEFICIARIES } \\
\hline & & FREQUENCY & PERCENTAGE & FREQUENCY & PERCENTAGE \\
\hline 1 & ILLITERATE & 17.00 & 28.33 & 26.00 & 43.33 \\
\hline 2 & $\begin{array}{c}\text { PRIMARY } \\
\text { SCHOOL } \\
\end{array}$ & 11.00 & 18.33 & 8.00 & 13.33 \\
\hline 3 & HIGH SCHOOL & 7.00 & 11.66 & 6.00 & 10.00 \\
\hline 4 & INTERMEDIATE & 8.00 & 13.33 & 6.00 & 10.00 \\
\hline 5 & $\begin{array}{c}\text { UNDER } \\
\text { GRADUATION }\end{array}$ & 8.00 & 13.33 & 6.00 & 10.00 \\
\hline 6 & $\begin{array}{c}\text { POST } \\
\text { GRADUATION }\end{array}$ & 9.00 & 15.00 & 8.00 & 13.33 \\
\hline & TOTAL & 60.00 & 100.00 & 60.00 & 100.00 \\
\hline
\end{tabular}




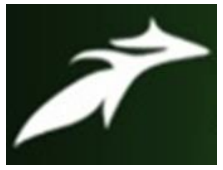

KOLA TIRUPATHI RAO et al, International Journal of Advances in Agricultural Science and Technology, Vol.7 Issue.11, November-2020, pg. 45-54

ISSN: 2348-1358 Impact Factor: 6.057 NAAS Rating: 3.77

Table no:3 Showing Family wise distribution of Beneficiaries and Non-Beneficiaries.

\begin{tabular}{|c|l|c|c|c|c|}
\hline S.NO & \multirow{2}{*}{ FAMILY } & \multicolumn{2}{|c|}{ BENEFICIARIES } & \multicolumn{2}{c|}{ NON BENEFICIARIES } \\
\cline { 3 - 6 } & TYPE & FREQUENCY & PERCENTAGE & FREQUENCY & PERCENTAGE \\
\hline 1 & NUCLEAR & 49.00 & 81.67 & 48.00 & 80.00 \\
\hline 2 & JOINT & 11.00 & 18.33 & 12.00 & 20.00 \\
\hline & & 60.00 & 100.00 & 60.00 & 100.00 \\
\hline
\end{tabular}

Table no: 4 Showing Family Size wise distribution of Beneficiaries and Non-Beneficiaries.

\begin{tabular}{|c|c|c|c|c|c|}
\hline \multirow[t]{2}{*}{ S.NO } & \multirow{2}{*}{$\begin{array}{l}\text { FAMILY } \\
\text { SIZE }\end{array}$} & \multicolumn{2}{|c|}{ BENEFICIARIES } & \multicolumn{2}{|c|}{ NON BENEFICIARIES } \\
\hline & & FREQUENCY & PERCENTAGE & FREQUENCY & PERCENTAGE \\
\hline 1 & $\begin{array}{c}\text { Upto } 5 \\
\text { MEMBERS }\end{array}$ & 35.00 & 58.33 & 34.00 & 56.7 \\
\hline 2 & $\begin{array}{c}\text { ABOVE } \\
\text { 5MEMBERS }\end{array}$ & 25.00 & 41.67 & 26.00 & 43.33 \\
\hline & & 60.00 & 100.00 & 60.00 & 100.00 \\
\hline
\end{tabular}

Table no:5 Showing Annual Income wise distribution of Beneficiaries and Non-Beneficiaries.

\begin{tabular}{|c|l|c|c|c|c|}
\hline \multirow{2}{*}{ S.NO } & \multirow{2}{*}{ INCOME } & \multicolumn{2}{|c|}{ BENEFICIARIES } & \multicolumn{2}{c|}{ NON BENEFICIARIES } \\
\cline { 3 - 5 } & & FREQUENCY & PERCENTAGE & FREQUENCY & PERCENTAGE \\
\hline 1 & Up to 40000 & 6.00 & 10.00 & 35.00 & 58.34 \\
\hline 2 & 40000 to 80000 & 16.00 & 26.67 & 21.00 & 35.00 \\
\hline 3 & Above 80,000 & 38.00 & 63.33 & 4.00 & 6.66 \\
\hline & 60.00 & 100.00 & 60.00 & 100.00 \\
\hline
\end{tabular}




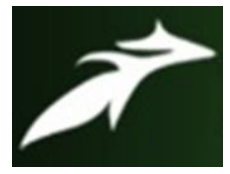

KOLA TIRUPATHI RAO et al, International Journal of Advances in Agricultural Science and Technology, Vol.7 Issue.11, November-2020, pg. 45-54

ISSN: 2348-1358 Impact Factor: 6.057 NAAS Rating: 3.77

Table no:6 Showing Land Holding wise distribution of Beneficiaries and Non-Beneficiaries.

\begin{tabular}{|c|l|c|c|c|c|}
\hline \multirow{2}{*}{ S. NO } & \multirow{2}{*}{$\begin{array}{c}\text { LAND } \\
\text { HOLDING }\end{array}$} & \multicolumn{2}{|c|}{ BENEFICIARIES } & \multicolumn{2}{c|}{ NON BENEFICIARIES } \\
\cline { 3 - 6 } & FREQUENCY & PERCENTAGE & FREQUENCY & PERCENTAGE \\
\hline 1 & Up to $1 \mathrm{Ha}$ & 13.00 & 21.67 & 37.00 & 61.67 \\
\hline 2 & $1-2 \mathrm{Ha}$ & 14.00 & 23.33 & 19.00 & 31.67 \\
\hline 3 & Above $2 \mathrm{Ha}$ & 33.00 & 55.00 & 4.00 & 6.66 \\
\hline & & 60.00 & 100.00 & 60.00 & 100.00 \\
\hline
\end{tabular}

Table no:7 Showing Livestock Possession wise distribution of Beneficiaries and Non-Beneficiaries.

\begin{tabular}{|c|c|c|c|c|c|}
\hline \multirow{2}{*}{ S.NO } & \multirow{2}{*}{$\begin{array}{l}\text { LIVESTOCK } \\
\text { POSSESSION }\end{array}$} & \multicolumn{2}{|c|}{ BENEFICIARIES } & \multicolumn{2}{c|}{ NON BENEFICIARIES } \\
\cline { 3 - 6 } & FREQUENCY & PERCENTAGE & FREQUENCY & PERCENTAGE \\
\hline 1 & Low & 10.00 & 16.66 & 19.00 & 31.67 \\
\hline 2 & Medium & 28.00 & 46.67 & 26.00 & 43.33 \\
\hline 3 & High & 22.00 & 36.67 & 15.00 & 25.00 \\
\hline & & 60.00 & 100.00 & 60.00 & 100.00 \\
\hline
\end{tabular}

Table no:8 Showing Mass Media Exposure wise distribution of Beneficiaries and Non-Beneficiaries.

\begin{tabular}{|c|c|c|c|c|c|}
\hline \multirow{2}{*}{ S.NO } & \multirow{2}{*}{\begin{tabular}{c} 
MASS MEDIA \\
\cline { 3 - 6 }
\end{tabular}} & \multicolumn{2}{|c|}{ EXPOSEFICIARIES } & \multicolumn{2}{c|}{ NON BENEFICIARIES } \\
\cline { 3 - 6 } & Low & 22.00 & 36.66 & 28.00 & 46.66 \\
\hline 1 & Medium & 28.00 & 46.66 & 24.00 & 40.00 \\
\hline 3 & High & 10.00 & 16.67 & 8.00 & 13.34 \\
\hline 3 & & 60.00 & 100.00 & 60.00 & 100.00 \\
\hline
\end{tabular}




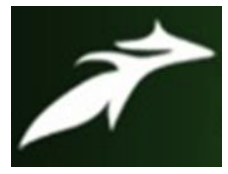

KOLA TIRUPATHI RAO et al, International Journal of Advances in Agricultural Science and Technology, Vol.7 Issue.11, November-2020, pg. 45-54

ISSN: 2348-1358

Impact Factor: 6.057

NAAS Rating: 3.77

Table no:9 Showing Extension Contact wise distribution of Beneficiaries and Non-Beneficiaries.

\begin{tabular}{|c|c|c|c|c|c|}
\hline S.NO & EXTENSION & \multicolumn{2}{|c|}{ BENEFICIARIES } & \multicolumn{2}{c|}{ NON BENEFICIARIES } \\
\cline { 3 - 6 } & CONTACT & FREQUENCY & PERCENTAGE & FREQUENCY & PERCENTAGE \\
\hline 1 & Low & 14.00 & 23.34 & 27.00 & 45.00 \\
\hline 2 & Medium & 19.00 & 31.66 & 17.00 & 28.34 \\
\hline 3 & High & 27.00 & 45.00 & 16.00 & 26.66 \\
\hline & & 60.00 & 100.00 & 60.00 & 100.00 \\
\hline
\end{tabular}

Table no:10. Knowledge wise distribution of the beneficiaries and non-beneficiaries towards Drip irrigation.

\begin{tabular}{|c|l|c|c|c|c|c|c|}
\hline \multirow{2}{*}{ S.NO } & \multirow{2}{*}{ KNOWLEDGE } & \multicolumn{3}{|c|}{ BENEFICIARIES } & \multicolumn{2}{c|}{ NON-BENEFICIARIES } \\
\cline { 3 - 8 } & & FC & PC & NC & FC & PC & NC \\
\hline 1 & WATER WAYS & $30(50)$ & $20(33.33)$ & $10(16.67)$ & $15(25)$ & $35(58.33)$ & $10(16.67)$ \\
\hline 2 & $\begin{array}{l}\text { PLOUGHING } \\
\text { ACROSS THE } \\
\text { SLOPE }\end{array}$ & $30(50)$ & $20(33.33)$ & $10(16.67)$ & $15(25)$ & $35(58.33)$ & $10(16.67)$ \\
\hline 3 & $\begin{array}{l}\text { LAND } \\
\text { SMOOTHENING }\end{array}$ & $30(50)$ & $20(33.33)$ & $10(16.67)$ & $12(20)$ & $16(26.67)$ & $32(53.33)$ \\
\hline 4 & $\begin{array}{l}\text { STRENGHENING } \\
\text { OF EXISTING } \\
\text { IRRIGATION }\end{array}$ & $25(41.67)$ & $25(41.67)$ & $10(16.67)$ & $15(25)$ & $35(58.33)$ & $10(16.67)$ \\
\hline 5 & $\begin{array}{l}\text { USE OF } \\
\text { IMPROVED } \\
\text { AGRICULTURAL } \\
\text { IMPLEMENT }\end{array}$ & $25(41.67)$ & $25(41.67)$ & $10(16.67)$ & $15(25)$ & $35(58.33)$ & $10(16.67)$ \\
\hline 6 & $\begin{array}{l}\text { INSTALLATION } \\
\text { OF DRIPPERS }\end{array}$ & $20(33.33)$ & $30(50)$ & $10(16.67)$ & $10(16.67)$ & $16(26.67)$ & $34(56.67)$ \\
\hline
\end{tabular}




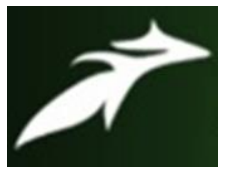

KOLA TIRUPATHI RAO et al, International Journal of Advances in Agricultural Science and Technology, Vol.7 Issue.11, November-2020, pg. 45-54

ISSN: 2348-1358

Impact Factor: 6.057

NAAS Rating: 3.77

\begin{tabular}{|c|c|c|c|c|c|c|c|}
\hline 7 & $\begin{array}{l}\text { MICRO SPRAY } \\
\text { HEADS }\end{array}$ & $10(16.67)$ & $35(58.33)$ & $15(25)$ & $10(16.67)$ & $15(25)$ & $35(58.33)$ \\
\hline 8 & $\begin{array}{l}\text { PROPER } \\
\text { WORKING OF } \\
\text { FILTERS }\end{array}$ & $15(25)$ & $30(50)$ & $15(25)$ & $10(16.67)$ & $14(23.33)$ & $36(60)$ \\
\hline 9 & $\begin{array}{l}\text { MOISTURE } \\
\text { WITHIN THE } \\
\text { ROOTZONE }\end{array}$ & $10(16.67)$ & $35(58.33)$ & $15(25)$ & $10(16.67)$ & $15(25)$ & $35(58.33)$ \\
\hline 10 & $\begin{array}{l}\text { INITIAL COST } \\
\text { MORE } \\
\text { COMPARED TO } \\
\text { OTHER SYSTEMS }\end{array}$ & $15(25)$ & $30(50)$ & $15(25)$ & $10(16.67)$ & $18(30)$ & $32(53.33)$ \\
\hline 11 & $\begin{array}{l}\text { KNOWLEDGE } \\
\text { REGARDING } \\
\text { DISTURBUTION } \\
\text { IN LINES }\end{array}$ & $15(25)$ & $30(50)$ & $15(25)$ & $10(16.67)$ & $18(30)$ & $32(53.33)$ \\
\hline
\end{tabular}

\begin{tabular}{|c|l|c|c|c|c|c|c|}
\hline \multirow{2}{*}{$\begin{array}{c}\text { S.N } \\
\text { O }\end{array}$} & \multirow{2}{*}{ KNOWLEDGE } & \multicolumn{3}{|c|}{ BENEFICIARIES } & \multicolumn{2}{c|}{ NON-BENEFICIARIES } \\
\cline { 3 - 7 } & & FC & PC & NC & FC & \multicolumn{2}{c|}{ PC } \\
\hline 1 & $\begin{array}{l}\text { Summer } \\
\text { ploughing }\end{array}$ & $12(20)$ & $38(63.33)$ & $10(16.67)$ & $15(25)$ & $35(58.33)$ & $10(16.67)$ \\
\hline 2 & $\begin{array}{l}\text { Across the } \\
\text { slope sowing }\end{array}$ & $17(28.33)$ & $30(50)$ & $13(21.67)$ & $17(28.33)$ & $25(41.67)$ & $18(30)$ \\
\hline 3 & $\begin{array}{l}\text { Improved } \\
\text { varieties }\end{array}$ & $16(26.67)$ & $30(50)$ & $13(21.67)$ & $15(25)$ & $30(50)$ & $15(25)$ \\
\hline 4 & Seed treatment & $18(30)$ & $32(53.33)$ & $10(16.67)$ & $10(16.67)$ & $25(41.67)$ & $25(41.67)$ \\
\hline 5 & Spacing & $12(20)$ & $32(53.33)$ & $16(26.67)$ & $10(16.67)$ & $20(33.33)$ & $30(50)$ \\
\hline 6 & $\begin{array}{l}\text { Application of } \\
\text { FYM }\end{array}$ & $18(30)$ & $32(53.33)$ & $10(16.67)$ & $10(16.67)$ & $25(41.67)$ & $25(41.67)$ \\
\hline 7 & Plant protection & $36(60)$ & $14(23.33)$ & $10(16.67)$ & $25(41.67)$ & $15(25)$ & $20(33.33)$ \\
\hline 8 & $\begin{array}{l}\text { Intercropping } \\
\text { techniques }\end{array}$ & $20(33.33)$ & $30(50)$ & $10(16.67)$ & $10(16.67)$ & $20(33.33)$ & $30(50)$ \\
\hline 9 & Fertilizers & $15(25)$ & $35(58.33)$ & $10(16.67)$ & $20(33.33)$ & $30(50)$ & $10(16.67)$ \\
\hline
\end{tabular}




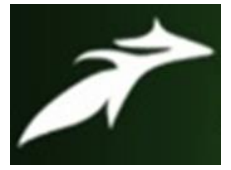

KOLA TIRUPATHI RAO et al, International Journal of Advances in Agricultural Science and Technology, Vol.7 Issue.11, November-2020, pg. 45-54

ISSN: 2348-1358

Impact Factor: 6.057

NAAS Rating: 3.77

Table no:11 Showing the Knowledge wise distribution of Beneficiaries and NonBeneficiaries of Drip irrigation.

\begin{tabular}{|c|l|c|c|c|c|}
\hline \multirow{2}{*}{ S.NO } & \multirow{2}{*}{ KNOWLEDGE } & \multicolumn{2}{|c|}{ BENEFICIARIES } & \multicolumn{2}{c|}{ NON BENEFICIARIES } \\
\cline { 3 - 5 } & & FREQUENCY & $\begin{array}{c}\text { PERCENTAG } \\
\text { E }\end{array}$ & FREQUENCY & $\begin{array}{c}\text { PERCENTAG } \\
\text { E }\end{array}$ \\
\hline 1 & Low(20-35) & 7.00 & 11.67 & 20.00 & 33.33 \\
\hline 2 & Medium(36-50) & 32.00 & 53.33 & 21.00 & 35.00 \\
\hline 3 & High(>50) & 21.00 & 35.00 & 19.00 & 31.67 \\
\hline & & 60.00 & 100.00 & 60.00 & 100.00 \\
\hline
\end{tabular}

Table no:12 Showing the Knowledge wise distribution of Beneficiaries and NonBeneficiaries in vegetable crop production.

\begin{tabular}{|c|c|c|c|c|c|}
\hline \multirow{2}{*}{ S.NO } & \multirow{2}{*}{ KNOWLEDGE } & \multicolumn{2}{|c|}{ BENEFICIARIES } & \multicolumn{2}{c|}{ NON BENEFICIARIES } \\
\cline { 3 - 5 } & & FREQUENCY & PERCENTAGE & FREQUENCY & PERCENTAGE \\
\hline 1 & Low (20-35) & 29.00 & 48.33 & 53.00 & 88.33 \\
\hline 2 & Medium(36-50) & 30.00 & 50.00 & 6.00 & 10.00 \\
\hline 3 & High(>50) & 1.00 & 1.67 & 1.00 & 1.67 \\
\hline & & 60.00 & 100.00 & 60.00 & 100.00 \\
\hline
\end{tabular}

Similar findings is also reported by Mondal, Shimul, Theerachai Haitook, and Suchint Simaraks. (2014): The data in the above table showed that most of the respondents 53.33 per cent having medium level and 35 per cent of respondents at high level \& 11.67 per cent of low level of respondents of knowledge in beneficiary(Drip irrigation) and 33.33 per cent of low $\& 35$ per cent of medium $\&$ 31.67 per cent of high was non beneficiaries (Drip irrigation) followed by 48.33 per cent of respondents belonged to low level of knowledge \& 50 per cent of respondents belongs to medium and 1 per cent of respondents from high level in beneficiary(vegetable crop production) \& 88.33 per cent of low \& 10 per cent of 


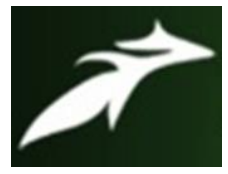

KOLA TIRUPATHI RAO et al, International Journal of Advances in Agricultural Science and Technology, Vol.7 Issue.11, November-2020, pg. 45-54

ISSN: 2348-1358 Impact Factor: 6.057 NAAS Rating: 3.77

respondents belongs to medium \& 1.67 per cent belongs to non beneficiary.(vegetable crop production).

\section{Correlation coefficient of profile characteristics of drip irrigation with their level of knowledge. $n=120$}

\begin{tabular}{|c|l|l|}
\hline Sl.no & \multicolumn{1}{|c|}{ Profile characteristics } & \multicolumn{1}{c|}{ "r" value } \\
\hline 1 & Age & $-0.063^{\mathrm{NS}}$ \\
\hline 2 & Education & $0.089^{* *}$ \\
\hline 3 & Family Size & $0.045^{* *}$ \\
\hline 4 & Family type & $0.064^{* *}$ \\
\hline 5 & Annual income & $0.038^{* *}$ \\
\hline 6 & Land holding & $0.004^{\mathrm{NS}}$ \\
\hline 7 & Livestock possession & $-0.021^{\mathrm{NS}}$ \\
\hline 8 & Mass media Exposure & $0.059^{* *}$ \\
\hline 9 & Extension Contact & $0.152^{* *}$ \\
\hline
\end{tabular}

$*=$ Significant at $\mathrm{p}=0.56, \mathrm{NS}=$ Non-significant

Similar findings is also reported by Bralts, Vincent F., I-Pai Wu, and Harris M. Gitlin(2005): The Data from the above table shows that education, family size and Extension contact are positively significant whereas age, land holding, livestock possession are non-significant was extend of knowledge of the respondents respectively. 


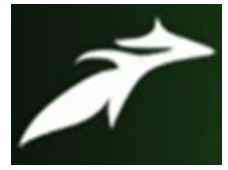

KOLA TIRUPATHI RAO et al, International Journal of Advances in Agricultural Science and Technology, Vol.7 Issue.11, November-2020, pg. 45-54

ISSN: 2348-1358

Impact Factor: 6.057

NAAS Rating: 3.77

\section{CONCLUSION:}

It was concluded that socio-economic profile of the beneficiaries were medium level whereas in case of non-beneficiaries were low level. The overall knowledge regarding drip irrigation in vegetable crop production in beneficiary had medium level of knowledge and non-beneficiary had low level of knowledge and relationship between knowledge level and socio-economic profile of respondents shows that Education, family size, Extension contact was positively significant at whereas Age, land holding and livestock possession was non-significant at extend of knowledge of respondents respectively.

\section{REFERENCES}

[1]. Bralts, Vincent F., I-Pai Wu, and Harris M. Gitlin(2005). "Manufacturing variation and drip irrigation uniformity." Transactions of the ASAE 24.1 : 113-0119.

[2]. Hartz, T. K., and G. J. Hochmuth. (1996): "Fertility management of drip-irrigated vegetables." HortTechnology 6.3 : 168-172.

[3]. Lamers, J., et al.(2014) "Anaerobic soil disinfestation for soil borne disease control in vegetable systems: current knowledge and future directions." VIII International Symposium on Chemical and Non-Chemical Soil and Substrate Disinfestation 1044.

[4]. Mondal, Shimul, Theerachai Haitook, and Suchint Simaraks. (2014) "Farmers' knowledge, attitude and practice toward organic vegetables cultivation in Northeast Thailand." Kasetsart J. Soc. Sci 35 : 158-166.

[5]. Marine, S. C., Martin, D. A., Adalja, A., Mathew, S., \& Everts, K. L. (2016). Effect of market channel, farm scale, and years in crop production on mid-Atlantic vegetable producers' knowledge and implementation of Good Agricultural Practices. Food Control, 59, 128-138.

[6]. Raine, S. R., et al.(2007) "Soil-water and solute movement under precision drip irrigation: knowledge gaps for managing sustainable root zones." Irrigation Science 26.1 : 91-100.

[7]. Simonne, Eric, et al. (2010): "Current knowledge, gaps, and future needs for keeping water and nutrients in the root zone of vegetables grown in Florida." HortTechnology 20.1 : 143-152. 